\title{
Los debates electorales en Twitter y su correspondencia con las preocupaciones ciudadanas en la contienda presidencial en Colombia 2018*
}

\author{
Electoral Debates on Twitter and How They Match the Citizen's Concerns about the 2018 Presidential Campaign \\ in Colombia \\ Os debates eleitorais no Twitter e sua correspondência com as preocupações cidadãs na corrida presidencial na \\ Colômbia 2018
}

Sergio Alvarado-Vivas ${ }^{\text {a }}$

Corporación Universitaria Minuto de Dios, Colombia

salvarado@uniminuto.edu

ORCID: https://orcid.org/0000-0001-6111-7550

DOI: https://doi.org/10.11144/Javeriana.syp39-76.detc

Juan López López

Universidad Santo Tomás, Colombia

Recibido: 06 Abril 2020

ORCID: https://orcid.org/0000-0002-5113-1524

Aceptado: 03 Agosto 2020

Joan Pedro-Carañana

Universidad Complutense de Madrid, España

ORCID: https://orcid.org/0000-0001-8958-0897

Publicado: 30 Diciembre 2020

\section{Resumen:}

El artículo se ocupa de cómo los temas más relevantes para la opinión pública colombiana se hicieron presentes en las discusiones y debates acaecidos en Twitter durante la campaña presidencial 2018. En un primer nivel, se analizó el grado de correspondencia entre los temas prioritarios para los ciudadanos, según las principales encuestas y la presencia y tratamiento axiológico de estos temas en Twitter, a partir de datos obtenidos mediante análisis de contenido. En un segundo nivel, se conjugaron datos cuantitativos y cualitativos que permitieron identificar la estructura de la racionalidad de la acción política que cada ideología promueve. Como resultado, no existió correspondencia alta entre los temas de los demoscópicos y las expresadas en Twitter. Además, las relaciones de poder por la hegemonía evidenciaron una predominancia de emisores de centro enfocados mayoritariamente en educación, mientras que la derecha y la izquierda con menos emisores lograron hegemonizar dos temas cada uno.

Palabras clave: Twitter, agenda pública, redes sociales, elecciones, Colombia.

\section{Abstract:}

This article delves into how the most relevant topics in the Colombian public opinion were present in the discussions and debates that took place on Twitter during 2018 presidential election campaign. At first, this work analyzes how those topics deemed as critical by the citizens, as stated in the main surveys, match the topics present and the axiological consideration thereof on Twitter. It is done based on data obtained from a content analysis. Secondly, quantitative and qualitative data were combined in order to identify the structure of rationality for the political action deployed by each ideological group. As a result, there was never a high correspondence between the demoscopic topics and those dealt with on Twitter. In addition, the power struggle to gain online hegemony showed a prevailing majority of central ideology users mainly focused on education, while the right and left groups with fewer users manage to impose two topics each group.

Keywords: Twitter, public agenda, social networks, elections, Colombia.

\section{Resumo:}

O artigo trata de como os temas mais relevantes para a opinião pública colombiana estiveram presentes nas discussões e debates acontecidos em Twitter durante a campanha presidencial 2018. Em um primeiro nível, analisou-se o grau de correspondência entre os temas prioritários para os cidadãos, de acordo com os principais inquéritos e a presença e tratamento axiológico destes temas no Twitter, a partir dos dados obtidos mediante análise de conteúdo. Em um segundo nível, conjugaram-se dados quantitativos e qualitativos que permitiram identificar a estrutura da racionalidade da ação política que cada ideologia promove. Como resultado,

Notas de autor

\footnotetext{
a Autor de correspondencia. Correo electrónico: salvarado@uniminuto.edu
} 
não houve grande correspondência entre os temas dos demoscópicos e os expressos no Twitter. Além disso, as relações de poder por hegemonia evidenciaram um predomínio de emissores de centro focados principalmente em educação, enquanto que a direita e a esquerda com menos emissores conseguiram hegemonizar dois temas cada uma.

Palavras-chave: Twitter, agenda pública, redes sociais, eleições, Colômbia.

\section{Introducción}

La pasada campaña presidencial colombiana de 2018 prometía ser histórica por ser la primera en su tipo con la participación de la otrora guerrilla FARC convertida en partido político ${ }^{[1]}$. No obstante, la prematura renuncia de su candidato oficial, Rodrigo, Timochenko, Londoño indicaba que, a diferencia lo acontecido en 2014, la discusión política en torno a la campaña iba más allá de los temas relacionados con el proceso de paz y el denominado "posacuerdo". De ahí que, en la presente investigación, se acudiera a estudios demoscópicos en busca de coordenadas que permitieran entender de modo más amplio la relación entre las preocupaciones ciudadanas y el debate político en curso.

A modo de contexto, también resulta relevante señalar que la contienda electoral contó con tres candidatos principales. Iván Duque del Centro Democrático representaba a la derecha, Gustavo Petro se presentó por Colombia Humana desde la izquierda y Sergio Fajardo concurrió con Alianza Verde como candidato de centro. En primera vuelta, Duque fue el candidato más votado (39,1\% de los votos) por delante de Petro $(25,1 \%)$, quien fue seguido muy de cerca por Fajardo $(23,7 \%)$. Duque se alzó con la victoria en segunda vuelta con un 53,98 \% de los votos frente al $41,81 \%$ de Petro.

\section{Objetivos}

El presente estudio investiga las narrativas que se compartieron en Twitter en torno a la campaña electoral, a partir de dos objetivos interrelacionados. El primero consiste en averiguar el grado de correspondencia entre los principales temas de preocupación de la opinión pública, según las encuestas más importantes y los temas y valores que se promueven en Twitter. Este objetivo permitirá alcanzar conclusiones sobre la representatividad del debate en esta red social respecto a las preocupaciones de la sociedad. El segundo objetivo consiste en medir la influencia de cada una de las tres ideologías principales de la modernidad (Bobbio, 2004; Galtung, 1992; Wallerstein, 2001) en los debates sobre cada tema clave, e identificar los contenidos concretos de las narrativas en lo referido a cómo relacionan a los candidatos con cada uno de estos temas. De este modo, se identifican y comparan las representaciones que ofrecen tuiteros de izquierda, de centro y de derecha sobre cada uno de los temas clave y se clasifican, siguiendo a Gramsci (2004), según sean hegemónicas, contrahegemónicas o minoritarias.

\section{Antecedentes}

La comunicación política y Twitter han llegado a tener una estrecha relación. En 2008, la campaña presidencial de Barack Obama marcó un hito al valerse de una estrategia de comunicación digital, dinamizada por ráfagas de microblogging de 140 caracteres. Esto no solo abrió un nuevo canal de comunicación entre el político y los ciudadanos, sino que sentó un precedente sobre el rol decisivo que pueden jugar las interacciones y los mensajes que circulan en los social media en la decisión del voto.

En términos de comunicación digital, la campaña de Obama estuvo orientada hacia la conformación de comunidades online, siendo los jóvenes los encargados de la activación direccionada de la movilización y la interacción entre usuarios. Mientras sus contrincantes seguían un número bastante reducido de cuentas 
(Gomes et al., 2009; López-Trigo et al., 2013), Obama optó por seguir a cientos de miles de usuarios (actualmente ronda los $600 \mathrm{mil}$ ). La idea fue mostrar interés por escuchar a las personas, conversar con ellas, ganarse su confianza y, de ese modo, atraer nuevos seguidores. Vale recordar que, como señalan Larsson y Moe (2012), en Twitter el seguimiento de otras personas se identifica principalmente con interés hacia otros porque, a diferencia de otras plataformas, el acto de seguir a un usuario en Twitter no es automáticamente recíproco. De ese modo, podría afirmarse que Obama y su campaña acertaron a autorrepresentarse en una clave de cercanía, apertura al diálogo y dependencia de la iniciativa de los usuarios para la consecución de su objetivo político.

Twitter no es solo una referencia clave para entender la experiencia política estadounidense, sino que también es fundamental para comprender la cultura política colombiana de los últimos años (Correa y Camargo, 2017; Rincón, 2011). En el caso colombiano, Twitter y Facebook hacen su aparición para las elecciones presidenciales de 2010. Tras el impedimento de la aspiración reeleccionista de Álvaro Uribe Vélez decretado por la Corte Constitucional, la opción de voto parecía abrirse a nuevas alternativas. Fue así como apareció Antanas Mockus, quien, en representación del Partido Verde, se constituyó en el candidato más novedoso en su forma de hacer campaña haciendo acopio de estrategias que, apoyadas en la adhesión masiva de la sociedad civil, lo llevaron hasta la segunda vuelta (Richard, 2011).

Como afirma Rincón, Mockus usó su cuenta sin intermediarios o representantes de su partido, y encontró en Twitter "su escenario predilecto para plantear temas, conocer qué pensaban sus seguidores y divulgar las ideas más problemáticas para atravesar los medios de comunicación” (2011, p. 81). A esto se sumó el fenómeno de la denominada \#OlaVerde, en la que simpatizantes de Mockus generaron un amplio abanico de formas creativas para exponer sus ideas a través de medios digitales. Sin embargo, los altos niveles de abstención, la maquinaría política y el músculo mediático de la campaña promovida por el uribismo inclinaron finalmente la balanza a favor de Juan Manuel Santos, quien luego de posesionarse tomó distancia de su padrino político.

El distanciamiento entre Álvaro Uribe y Juan Manuel Santos definió cuatro años después la siguiente contienda presidencial. Santos, que buscaba un segundo mandato, tuvo como adversario principal a Óscar Iván Zuluaga, candidato del Centro Democrático ${ }^{[2]}$. Exponiendo diferentes visiones, ambos candidatos convirtieron el proceso de paz con las FARC en el principal tema de discusión en los debates. Entretanto, Twitter abrigó confrontaciones abiertas, incluso agresivas, en las que participaban candidatos, políticos, ciudadanos, periodistas y equipos de campaña por igual. Dichas polémicas estuvieron motivadas por eventos y actores variados, desde escándalos por hackers que espiaban las negociaciones de La Habana hasta pautas publicitarias que para muchos daban cuenta de una desafortunada campaña de odio promovida por el Centro Democrático (García-Perdomo, 2017). Finalmente, la tensión y el conflicto se intensificaron con la derrota de Zuluaga, pues en los años que siguieron el Centro Democrático se empeñó aún más en promover una lectura de país seriamente amenazado por la confabulación de dos antagonistas principales: las FARC y el presidente Juan Manuel Santos (Cifuentes y Pino, 2018).

En la actualidad, los estudios de comunicación política y Twitter evidencian tendencias de investigaciones relacionadas con la predicción electoral (Jungherr et al., 2015), los usos que le dan los ciudadanos y candidatos (además de los partidos que representan) (Caldevilla et al., 2019; Castromil et al., 2020), los impactos en los resultados electorales ( $\mathrm{Su}$ y Borah, 2019), la viralidad de lo trinado y el análisis de los sentimientos que se construyen a través de los flujos digitales de quienes interactúan (Percastre-Mendizábal et al., 2017). Se suman también perspectivas de análisis basadas la clusterización (Jara et al., 2017), el framing (GarcíaPerdomo, 2017), el análisis del discurso y el análisis de contenido (Percastre-Mendizábal et al., 2017). Se trata de un nutrido árbol de investigaciones que toman a Twitter como un magnífico laboratorio para poner a prueba modos alternativos de examinar las contiendas políticas contemporáneas (Campos-Domínguez, 2017; Fernández, 2012). 
Si bien el número de estudios a nivel global va en crecimiento, en Colombia, las investigaciones empíricas que relacionan campañas electorales y Twitter siguen siendo escasas. No obstante, sí que cabe reseñar algunos que se inscriben en el ámbito más general de la relación entre política, comunicación y social media. Textos como el de Richard (2011), Rincón (2011) y García-Dussán (2011) hacen hincapié en las estrategias y novedades de lo digital en las campañas durante las elecciones presidenciales de 2010, y enfatizan en el fenómeno \#OlaVerde y la candidatura de Antanas Mockus. Investigaciones como las de Said-Hung y Arcila (2011) o Segado-Boj et al., (2015) se concentran en los líderes de opinión o líderes políticos con mayor visibilidad en Twitter, sus rasgos principales, las características de sus mensajes y sus formas de reaccionar en momentos de crisis. También hay un conjunto de estudios sobre la polarización electoral, el proceso de paz con las FARC, la consolidación de la derecha en Colombia y la figura de Álvaro Uribe como configurador de la bancada del Centro Democrático (Bonilla y Velandia, 2010; Cifuentes y Pino, 2018; Gallego et al., 2017; García-Perdomo, 2017; Mejía Cortés, 2018). Finalmente, muy en la línea de lo que se presenta aquí, hay que mencionar el texto de Quintero y Giraldo (2018), donde se explora la relación entre estudios demoscópicos y los debates que tienen lugar en Twitter.

\section{Metodología}

El proceso metodológico llevado a cabo en la presente investigación consta de tres partes diferenciadas. En primer lugar, se identifican los temas prioritarios para los ciudadanos a partir de la revisión de estudios demoscópicos. En segundo lugar, se explica el procedimiento que se ha llevado a cabo para la selección de la muestra de tuits que son pertinentes y tienen una incidencia destacada en Twitter. La tercera parte presenta el modelo de análisis de contenido que se ha aplicado a cada unidad muestral.

\section{Revisión de estudios demoscópicos}

Dado que la campaña presidencial transcurrió entre marzo y junio de 2018, se decidió rastrear sondeos demoscópicos que a nivel nacional estuvieran midiendo la opinión pública alrededor de la elección del presidente para el periodo 2018-2022. Esto desembocó en la identificación de dos sondeos de referencia, uno realizado por la firma YanHaas Advanced Market Research $(2018)^{[3]}$ y el otro realizado por Investigación y Asesoría de Mercadeo [Invamer] (2018) ${ }^{[4]}$. En cada una de las encuestas, se seleccionaron preguntas centradas en los temas importantes y prioritarios para los votantes a ser considerados por el próximo presidente. Como resultado, se filtraron cinco temas recurrentes como los más relevantes en ambos estudios: salud, educación, desempleo, corrupción e inseguridad (Tabla 1$)^{[5]}$.

TABLA 1.

Resultados de los estudios demoscópicos de mayo 2018 de Yanhaas e Invamer

\begin{tabular}{|c|c|}
\hline $\begin{array}{c}\text { YanHaas } \\
(\%)\end{array}$ & $\begin{array}{c}\text { Invamer } \\
(\%)\end{array}$ \\
\hline $\begin{array}{l}\text { ¿Qué tan importante es ... para tomar su decisión sobre } \\
\text { el candidato presidencial por el cual votar? }\end{array}$ & $\begin{array}{l}\text { ¿Cuál es el principal problema que debe ser resuelto } \\
\text { por el próximo presidente de Colombia? }\end{array}$ \\
\hline Salud (Muy importante 90) & La calidad y el cubrimiento de la salud $(19,2)$ \\
\hline Educación (88) & Corrupción $(18,8)$ \\
\hline Seguridad (81) & Desempleo $(16,2)$ \\
\hline Desempleo (80) & La calidad y el cubrimiento de la educación $(12,2)$ \\
\hline Medio Ambiente (79) & Inseguridad $(8,2)$ \\
\hline Corrupción (76) & Pobreza $(5,6)$ \\
\hline
\end{tabular}

Fuente: elaboración propia. 


\section{Selección y refinamiento de la muestra}

El rango de fechas establecido para el rastreo y extracción de tuits fue del 12 de marzo al 18 de junio de 2018. La primera fecha hace referencia al día después de la realización de las elecciones al Senado y Cámara de representantes, momento que marcó el arranque de las campañas presidenciales. La fecha de cierre de la muestra se refiere al día después de las elecciones, momento en el que se da por finalizada la actividad en Twitter en torno a las elecciones.

Para una recogida sistemática de los tuits se estableció como un primer parámetro la selección de Trending Topics (TT) que tuvieran relación estrecha con la campaña electoral presidencial. Indistintamente, si se trataba de un hashtag creado por un candidato, por un líder de opinión o un ciudadano del común aquí lo importante era su ranking en los TT del día. Para tal fin, se recurrió al uso de la web Trendinalia.com, una plataforma que permite rastrear diariamente los TT más potentes a nivel mundial, ofreciendo también la posibilidad de realizar un filtro por país. Esta opción resultó conveniente para delimitar el listado de TT al ámbito colombiano.

Dado que Trendinalia arroja en promedio un listado de 200 TT por día, clasificados por número de horas de presencia a lo largo del día, se decidió, como un segundo parámetro, tener en cuenta únicamente los TT relacionados con la campaña electoral que estuvieran en los primeros diez lugares de los resultados. De este modo, se dio prioridad a los hashtags más influyentes, lo que en tiempo representa una duración de al menos siete $\mathrm{u}$ ocho horas de presencia en Twitter.

De cada TT, se decidió recoger un lote de 10 trinos, los cuales fueron seleccionados directamente de la web de Twitter a través de la pestaña denominada Destacados, donde se muestran tuits que están soportados por cifras significativas de interacción traducidos en favs, retweets . replies. Adicionalmente, para evitar contaminaciones, se hizo la extracción sin iniciar sesión desde alguna cuenta de usuario. Con ello, se pretendía evitar que la opción de Destacados se ajustase a las preferencias particulares de cualquier tipo. Fue así como se logró construir una muestra de 620 tuits producto de 62 TT identificados a lo largo de las 14 semanas en que tuvo lugar la campaña electoral.

Posteriormente se realizó un refinamiento de la muestra recurriendo al uso de filtros para extraer del total de la muestra los trinos alusivos a alguno de los cinco temas identificados en las encuestas YanHaas (2018) e Invamer (2018). Se buscó tanto la aparición literal del concepto como una serie de keywords relacionadas con cada tema para realizar la búsqueda de la presencia de los temas en los trinos Destacados hallados en Twitter (Tabla 2).

TABLA 2.

Keywords empleadas para la selección de tuits.

\begin{tabular}{|c|c|c|}
\hline Tema & Keywords empleadas para la selección de tweets & N. ${ }^{\circ}$ de menciones \\
\hline 1 Salud & Seguridad social, hospital, Sisbén, medicina, EPS & 8 \\
\hline 2 Educación & $\begin{array}{l}\text { Estudio, estudia, pedagogia, primaria, secundaria, bachillerato, } \\
\text { universidad, SENA, escolaridad, educar, enseñar }\end{array}$ & 60 \\
\hline 3 Desempleo & Empleo, trabajo, trabajadores, laboral, salario & 27 \\
\hline 4 Corrupción & $\begin{array}{l}\text { Corrupto, legalidad, ilegalidad, mermelada, honestidad, honradez, } \\
\text { anticorrupción, transparencia, robar, delitos, procesos judiciales, leyes, } \\
\text { mafias }\end{array}$ & 56 \\
\hline \multirow[t]{2}{*}{5 Seguridad } & $\begin{array}{c}\text { Seguridad, ladrón, ladrones, cárcel, miedo, temor, delincuencia, } \\
\text { violencia, robos }\end{array}$ & 25 \\
\hline & Total & 176 \\
\hline
\end{tabular}

Fuente: elaboración propia.

\section{Método de análisis de contenido}

Se aplicó un análisis de contenido sistemático y estructural a cada uno de los tuits seleccionados (unidades de la muestra). A partir del modelo de la mediación cognitiva elaborado por Martín Serrano (2004), se elaboró 
un protocolo de análisis de contenido que incluía categorías de análisis tanto cuantitativas como cualitativas y que permitió vincular tres variables fundamentales de las narrativas: las características del emisor, la lógica de los actos de los actores protagonistas en el relato y el marco axiológico que propone el emisor. Además, el protocolo de análisis permitió cuantificar el número de tuits pertinentes que contenía cada TT. A fin de identificar los emisores y su presencia en el debate sobre la campaña electoral en Twitter, el protocolo de análisis se diseñó de tal forma que se pudiera codificar la ideología del emisor a partir de tres opciones: izquierda (1), centro (2) y derecha (3).

La segunda variable de análisis permitió obtener datos cuantitativos y cualitativos sobre los actores protagonistas, los roles desempeñados, las características atribuidas, los actos llevados a cabo, los medios o instrumentos empleados para ello (cómo lo hacen), los objetivos que se perseguían (para qué) y si su consecución termina en logro (1) o fracaso (2). además, se recogen los actores secundarios con los que interactúa el actor protagonista y si el tipo de relación es de asociación (1) o de oposición (2). Este análisis permite distinguir los actores según su nivel de presencia, recoger la forma en que han sido descritos en los tuits e identificar la estructura de la racionalidad de la acción política que proponen los mediadores sobre cada tema clave por parte de cada actor protagonista.

Por último, se buscaba identificar el marco axiológico de los tuiteros. Para ello, se investiga si el actor y la lógica de sus actos son evaluados de manera positiva (1) o negativa (2), así como los valores que se defienden (honestidad, justicia, seguridad...) y del tono que se emplea (informativo, elogioso, de denuncia...).

\section{Resultados}

\section{Objetivo 1: Grado de correspondencia entre preocupaciones ciudadanas y preocupaciones en Twitter}

De la muestra total de 62 hashtags relacionados con la campaña presidencial que lograron ser Trending Topic, $46(74,2 \%)$ de ellos contienen al menos un tuit que aborda alguno de los cinco temas de interés ranqueados por las encuestas. Esto indica una presencia media-alta de TT que tratan alguno de los temas prioritarios.

El dato más relevante es la presencia de 176 menciones de keywords sobre los temas prioritarios $(28,4 \%$ de los 620 trinos) en 145 tuits (23,4\%), que se interpreta como una correspondencia media-baja. Estas cifras indican que los debates de Twitter no están dominados por los temas clave de los estudios demoscópicos.

En cuanto a la correspondencia entre los temas prioritarios para los ciudadanos según los estudios de Invamer (2018) y YanHaas (2018) y el orden de importancia de estos cinco temas en Twitter en función del número de menciones, se observa un grado de correspondencia que puede definirse como medio-alto (Tabla 3).

TABLA 3.

Resultados estudios demoscópicos versus número menciones en Twitter por tema.

\begin{tabular}{cccc}
\hline Tema & $\begin{array}{c}\text { ¿Qué tan importante es (...) para } \\
\text { tomar su decisión sobre el candidato } \\
\text { presidencial por el cuál votar? } \\
\text { (Yanhaas) } \\
\text { (\%) }\end{array}$ & $\begin{array}{c}\text { iCuál es el principal problema } \\
\text { que debe ser resuelto por el } \\
\text { próximo presidente de Colombia? } \\
\text { (Invamer) } \\
\text { (\%) }\end{array}$ & $\begin{array}{c}\mathbf{N} . \text { de }^{\circ} \\
\text { menciones en } \\
\text { Twitter (\%) }\end{array}$ \\
\hline Salud & Muy importante 90 & 19,2 & $8(4,5)$ \\
\hline Educación & 88 & 12,2 & $60(34)$ \\
\hline Desempleo & 80 & 16,2 & $27(15,3)$ \\
\hline Corrupción & 76 & 18,8 & $56(31,8)$ \\
\hline Seguridad & 81 & 8,2 & $25(14,2)$ \\
\hline & & Total & 176 \\
\hline
\end{tabular}

Fuente: elaboración propia.

Salud resulta ser el tema más disímil ya que si bien es el que encabeza en los estudios demoscópicos, en Twitter su realidad es otra, apenas alcanzando el 4,5\% (ocho menciones), relegándolo a la última plaza de los cinco temas. También puede observarse una ligera discrepancia en el tema Corrupción, pues ocupa el cuarto 
lugar en las encuestas mientras que en Twitter se alza con la segunda posición con 56 presencias $(31,8 \%)$. Los otros temas presentan una correspondencia alta.

Cabe señalar otro resultado que muestra coherencia entre los datos de las encuestas y los de Twitter. Aunque el TT \#YLaSeguridadQue ${ }^{[6]}$ resultó ser la etiqueta que más menciones aporta (12), la Seguridad es el penúltimo tema en presencia en la red social en función de las menciones a conceptos explícitamente relacionados con la seguridad: robos, miedo, delincuencia..., y el último en el ranking de las encuestas. Esto es debido a la presencia de otros temas prioritarios bajo este hashtag, lo que indica un cambio en la representación de la seguridad, apareciendo vinculada a educación, salud, empleo y corrupción, temas que los encuestados consideran más importantes que la seguridad frente al crimen. Si bien el tema Seguridad ha sido hegemonizado por la derecha, los tuiteros de centro e izquierda han remarcado los vínculos con otros temas. De hecho, esta etiqueta se genera a partir de la entrevista al candidato de izquierda Gustavo Petro en Caracol Noticias sobre temas de seguridad.

Respecto al marco axiológico de los tuits, se observa, en primer lugar, una coherencia alta entre los valores defendidos por quienes emiten los trinos y la mejora de los temas clave identificados por los ciudadanos en las encuestas. Se halló un espectro amplio donde destaca la defensa de la equidad (26,1\%), la cual abarca aspectos como el acceso a la educación, condiciones justas para los ciudadanos y procesos de inclusión en diferentes niveles. Otros valores a destacar, que además suelen aparecer vinculados con la equidad, son la honestidad y la lucha anticorrupción (17\%) y la capacidad de criticidad y autonomía $(8,5 \%)$. También aparecen defensas de la laboriosidad $(7,4 \%)$ como un valor determinante a la hora de votar por un candidato u otro, además de la legalidad $(6,9 \%)$ y la coherencia $(6,9 \%)$ en el proceder de los políticos.

En segundo lugar, destaca que predominan el tono informativo con un 31,4\% y el elogioso con un 29,5\%. Le siguen con un importante rezago el tono conciliador/mediador $(15,9 \%)$, mientras que tonos como el de protesta/denuncia (11,6\%), el descalificador/ridiculizador (6,6\%) y el ofensivo (3,9\%) tienen una presencia no demasiado significativa pese a tratarse de debates ciudadanos en plena contienda electoral. La priorización de Tonos positivos se interpreta como congruente con la consecución de objetivos vinculados a los temas clave para los ciudadanos, pues son temas que afectan a la mayor parte de la población y que requieren difusión de información, propuestas concretas (a menudo las propuestas de los candidatos, que son elogiados por ellas) y conciliación y unidad entre los colombianos por el bien común. La mera protesta expresiva respecto a estos temas de interés nacional no serviría para mejorar, pero un enfoque propositivo tiene un carácter instrumental orientado a incidir positivamente en la resolución de problemas vinculados a los temas clave. Estos resultados son coherentes con un predominio de evaluaciones positivas de los actores y sus actuaciones $(75 \%)$.

\section{Objetivo 2: Análisis de la estructura de la acción política según emisor y tema}

En este apartado se presentan los resultados del análisis de contenido sobre la estructura de los relatos de cada tema clave en función de si sus emisores tienen una ideología de izquierda, de centro o de derecha, y se clasifican según sean, en Twitter, hegemónicos, contrahegemónicos o minoritarios. De este modo, se profundiza en las características de los discursos en Twitter según emisores e influencia. Además de las diferencias entre los relatos, conviene señalar que las narrativas son similares en términos de que en todas ellas hay una mayor presencia de evaluaciones, tonos y valores positivos que negativos (en concordancia con los hallazgos cuantitativos de la visión de conjunto).

A nivel del total de la muestra, destaca que el centro emite la mayor parte de tuits con un $42 \%$, seguido con algo de distancia por la izquierda $(30,1 \%)$ y la derecha $(27,8 \%)$. No obstante, si se atiende la influencia de cada una de las ideologías en los discursos sobre cada uno de los temas clave se observa que la izquierda hegemoniza dos temas (salud y corrupción), el mismo número que la derecha (desempleo e inseguridad), mientras que el centro controla el debate solamente en el tema educación (Tabla 4). 
TABLA 4.

Presencia de los emisores según tema y orientación ideológica.

\begin{tabular}{|c|c|c|c|c|c|c|c|c|c|c|c|c|c|c|c|}
\hline Temas & & Salud & & & ducaci & & & esemple & & & isegurid & & & orrupci & \\
\hline $\begin{array}{l}\text { Orientación } \\
\text { Ideológica }\end{array}$ & Izq. & Cen. & Der. & Izq. & Cen. & Der. 1 & Izq. $\mathrm{C}$ & Cen. I & Der. 1 & Izq. & Cen. & Der. & Izq. & Cen. & Der. \\
\hline Total tuits & 4 & 3 & 1 & 15 & 45 & 1 & 7 & 4 & 16 & 7 & 4 & 16 & 20 & 18 & 15 \\
\hline $\begin{array}{c}\text { Total tuits } \\
(\%)\end{array}$ & 2,3 & 1,7 & 0,6 & 8,5 & 25,6 & 0,6 & 4,0 & 2,3 & 9,1 & 4,0 & 2,3 & 9,1 & 11,4 & 10,2 & 8,5 \\
\hline $\begin{array}{c}\text { Total por tema } \\
(\%)\end{array}$ & & 4,5 & & & 34,7 & & & 15,3 & & & 15,3 & & & 30,1 & \\
\hline
\end{tabular}

Fuente: elaboración propia.

Estos datos se utilizan para organizar las mediaciones de cada ideología según tengan una posición hegemónica, contrahegemónica o minoritaria. Se deduce que la derecha, seguida de la izquierda, han sido capaces de hegemonizar más temas con menos tuits totales. La eficacia global del centro se ve reducida por concentrarse en el tema educación.

También es relevante que destacan hashtags vinculados a Fajardo, como \#UnPresidenteProfesor (10 menciones a temas clave), \#FajardoEnUnivalle (9), \#FajardoLogró (8) y \#YoVotoFajardo (8). Si bien un mayor control de los TT otorga visibilidad, el dominio de Fajardo a este respecto no se traduce en una mayor hegemonía del centro en cuanto al número de temas, en consonancia con los datos de los emisores. Se ha encontrado una influencia similar en el tema salud de la izquierda con cuatro participaciones y del centro con tres. La derecha ocupa una posición minoritaria con solo un trino.

La narrativa de izquierda se sintetiza de la siguiente manera: el candidato (rol) Gustavo Petro (actor protagonista), como defensor de la administración pública (atributos), propone un sistema de salud público (actos), que combine la prestación de servicios públicos, privados y mixtos (medios), a fin de dignificar a las personas y mejorar su bienestar y empleabilidad, especialmente de aquellas en condición de discapacidad (objetivos). La defensa de la salud pública se hace en oposición (interacción) al centro y a la derecha (actores secundarios) por no querer realizar cambios sustanciales y por haber impulsado un "carrusel de la muerte" ${ }^{\text {[7] }}$, respectivamente. Los tuiteros defienden, principalmente, el valor de la equidad, aunque también la solidaridad.

La mediación cognitiva de centro se refiere en dos ocasiones a Humberto de la Calle y una vez a Sergio Fajardo (actores protagonistas). El candidato ( $\mathrm{rol}$ ) de la calle es representado como promotor del cambio en salud (atributos), impulsando la medicina preventiva y proponiendo políticas antidrogas (actos), basadas en el sistema de salud público frente al negocio de la industria (medios). Se argumenta que de este modo podrá mitigarse el consumo de drogas en el país y mejorar la salud de los colombianos (objetivos). La reforma se hará en oposición (interacción) a las propuestas prohibitivas y punitivas de Iván Duque (actor secundario). por su parte, el candidato (rol) fajardo es presentado como un político sensato (atributos) que no quiere hacer giros bruscos sin una planeación adecuada del sistema de salud (actos) y, por tanto, no acabará abruptamente las EPS (medios). Así, Fajardo combate el populismo (objetivo) y se opone (interacción) a las propuestas y formas de administrar de Gustavo Petro (actor secundario). Se defienden los valores de la equidad con responsabilidad.

La derecha está autorrepresentada por el candidato (rol) Germán Vargas Lleras (actor protagonista), quien se caracteriza por ser servicial y ambicioso (atributos). Afirma proponer el programa más ambicioso en términos de salud, pensiones y empleo (actos) para transformar el país (objetivos). Cabe destacar que no hay ninguna narrativa favorable al vencedor de las elecciones, Iván Duque. El tema educación está ampliamente hegemonizado por una mediación favorable al centro ( 45 tuits). En segundo lugar y a mucha distancia está la izquierda (15 tuits). La derecha ocupa una posición minoritaria con solo un tuit.

La mediación cognitiva hegemónica se sintetiza de la siguiente manera: el profesor y candidato (rol) Sergio Fajardo (actor protagonista) está plenamente preparado (atributos) para hacer de la educación un gran proyecto nacional de mejora de las oportunidades educativas, la calidad y el desempeño escolar (actos), mediante un incremento de la inversión proveniente de fondos de la lucha anticorrupción (becas, salarios, dotación de infraestructura), una mejor preparación docente y un aumento de los cupos (medios). Esta 
reforma educativa permitirá a los colombianos convivir en un futuro digno que reduzca la vulnerabilidad de los niños, con mayores oportunidades para las mujeres y orientado al desarrollo sostenible (objetivos). todo ello se hará en asociación (interacción) con los estudiantes y los profesores (actores secundarios). La experiencia previa de Fajardo se presenta como prueba de que estos objetivos serán logrados. destaca ampliamente el valor de la equidad, seguido de la coherencia.

La narrativa de izquierda comparte similitudes con la de Fajardo. Según los tuits, el profesor y candidato (rol) Petro (actor) también está altamente cualificado (atributos) para llevar a cabo un gran proyecto educativo (acto) basado en mayor inversión, más calidad y mejores infraestructuras (medio). Sin embargo, se diferencia por realizar una propuesta de mayor alcance como es la gratuidad de la educación superior (medio). En cuanto a los objetivos sociales que persigue la reforma educativa de Petro, destaca que permitirá reducir la delincuencia al facilitar mayores oportunidades y la inclusión social. En segundo lugar, la educación también se vincula con la construcción de paz. La reforma educativa también se llevará a cabo en asociación (interacción) con la comunidad educativa (actor secundario). Los tuits se alinean con el valor de la equidad.

El único trino de derecha critica que la rectora de la Universidad del Atlántico (actor protagonista) haya alabado al excomandante de las FARC Jesús Santrich (actos) en un discurso público (medios), con el propósito de confundir a la juventud (objetivos). El tuitero evalúa negativamente esta acción en un tono de protesta y defiende el valor de la responsabilidad.

La discusión sobre empleo está hegemonizada por los bloques de derecha con 16 trinos. Le siguen de lejos la izquierda con siete participaciones y el centro con cuatro. La narrativa hegemónica se refiere a Iván duque (actor protagonista) como la alternativa tanto a las políticas de Juan Manuel Santos como de Gustavo Petro (rol), por ser un político realista y eficaz (atributos). Según esta narrativa, Duque impulsará el empleo, especialmente en el sector agrícola, la competitividad, la inversión, las exportaciones, la formalización del trabajo, los salarios y el emprendimiento (actos) mediante una reforma tributaria que permita bajar los impuestos y agilizar los trámites burocráticos (medios). Todo ello redundará en un mayor crecimiento económico y evitará el auge del populismo y que Colombia se convierta en la nueva Venezuela (objetivos). Las propuestas de Duque se oponen (interacción), especialmente, a las del populista Gustavo Petro (actor secundario). Predomina el valor de la laboriosidad.

El relato de izquierda identifica a Gustavo Petro (actor protagonista) como un candidato alternativo al establishment (rol), caracterizado por su austeridad (atributo). Plantea la creación de un sistema de créditos asequibles, la revitalización del campo, la mejora del empleo y el incremento de los salarios (actos), mediante una política estatal de gasto público y la derogación de la reforma laboral implementada por Álvaro Uribe (medios). De este modo, se logrará desarrollo y la economía beneficiará a los ciudadanos, quienes podrán tener un futuro en Colombia (objetivos). La mejora del empleo podrá realizarse en oposición (interacción) a las fuerzas de derechas (actor secundario). Esta narrativa da prioridad al valor de la equidad.

La visión minoritaria del centro identifica al candidato (rol) Sergio Fajardo (actor protagonista) como el adalid contra la corrupción (atributos). Se expone que Fajardo mejorará la economía y el empleo (actos), mediante la lucha anticorrupción, la educación, el fomento de proyectos productivos y la atención a zonas remotas (medios). Las reformas permitirán a todos los ciudadanos tener un futuro (objetivo). Se argumenta que este objetivo será logrado porque Fajardo ya lo consiguió como alcalde de Medellín y gobernador de Antioquia. Se defiende el valor de la equidad. El debate en torno a seguridad está hegemonizado por la derecha, con 16 tuits. La izquierda cuenta con siete tuits y el centro con cuatro.

El relato hegemónico se refiere a los candidatos (rol) Iván Duque y Germán Vargas Lleras (actores protagonistas). Los dos candidatos de partidos de derechas son caracterizados como políticos muy preparados y duros con la delincuencia (atributos), que prometen reducir el crimen (actos), con un aumento de la fuerza policial, las medidas punitivas, la lucha contra guerrilleros y la inversión económica (medios). La lucha contra la delincuencia no solo se llevará a cabo en oposición (interacción) a los grupos criminales, sino también a 
Gustavo Petro (actor secundario) por querer debilitar a las fuerzas de seguridad. El valor principal es el de la legalidad, aunque también está presente la equidad.

La izquierda presenta al candidato (rol) Gustavo Petro (actor protagonista) como un político sin vínculos con grupos criminales ni con corruptos (atributos). Petro propone combatir la delincuencia (actos) mediante la reducción de la desigualdad, la educación, las políticas de inclusión social de los jóvenes y mecanismos efectivos de defensa de los líderes sociales y no mediante la construcción de más cárceles ni medidas represivas (medios). Petro se opone (interacción) a las políticas y apoyos de Iván Duque (actor secundario). Se promueven los valores de equidad y honestidad.

El centro ve en el candidato (rol) Sergio Fajardo (actor protagonista) el político capaz de promover el paso del miedo a la esperanza (actos), a través de la apropiación ciudadana del espacio público y mayores oportunidades para los guerrilleros (medios), como ya hizo en Antioquia (logro). Fajardo mejorará la seguridad en asociación (interacción) con los ciudadanos, especialmente la comunidad educativa (actores secundarios). El candidato (rol) Humberto de la Calle (actor protagonista) tiene la misma presencia que Fajardo con dos menciones. Se dice que impulsará la seguridad (actos), con políticas públicas de inclusión y tratamiento médico (medios) en oposición (interacción) a las políticas represivas y propuestas agrarias feudales de Iván Duque (actor secundario) que solamente generan más violencia. Se promueven los valores de equidad y honestidad. En el tema corrupción, las tres ideologías están bastante parejas. La izquierda emite 20 trinos, el centro 18 y la derecha 15.

La visión hegemónica de la izquierda identifica al candidato (rol) Gustavo Petro (actor protagonista) como el actor que no tiene deudas con la justicia ni maquinaria electoral y el abanderado de la lucha contra la corrupción (atributos). La narrativa indica que Petro promueve la unidad frente a la corrupción (actos) mediante la memoria y la conciencia histórica (medios). Esta actuación debe llevarse a cabo en oposición (interacción) a Uribe y todos los corruptos y en asociación (interacción) con ciudadanos libres y conscientes. la lucha anticorrupción se vincula con la justicia y la paz (objetivos). El discurso de izquierda da prioridad a los valores de la honestidad y la autonomía.

En el relato contrahegemónico, el centro define al candidato (rol) Sergio Fajardo y Claudia López (actores protagonistas) como honestos, decentes, transparentes, eficientes y sin maquinaria electoral ni ánimo de revancha (atributos). Se dice que los candidatos de centro promueven la unidad para recuperar un $1 \%$ del PIB que se pierde por corrupción (actos), mediante el respeto a la ley y la educación (medios). Los fondos obtenidos se dedicarán al empleo, la inversión en educación, tecnología y cultura, a la paz y a la equidad (objetivos). Se actuará en asociación (interacción) con todas las personas honradas y en oposición (interacción) a los corruptos. Se priorizan los valores de honestidad, autonomía y legalidad.

En el seno de la derecha, la visión dominante alaba del candidato (rol) Iván Duque (actor protagonista) su carácter firme (atributos) para enfrentar la corrupción (actos), mediante la ley y la seguridad (medios). Así podrá impulsarse el emprendedurismo y reordenar la economía del país (objetivos). La lucha anticorrupción se hará en oposición (interacción) al chavista Petro, quien se ha forjado en el crimen organizado (M19), es ineficiente y despilfarrador, pero también a Vargas Lleras por representar la continuidad del gobierno "enmermelado" de Santos. Por su parte, la campaña del candidato (rol) Lleras (actor protagonista) le autodefine como un político limpio y de mano dura contra la corrupción (atributos), que aplicará las normas existentes (actos) mediante la dotación de más jueces y recursos (medios), en oposición (interacción) al partido de Duque que recibe apoyos de corruptos (actor secundario). En ambos casos, se alude a los valores de legalidad, honestidad y autonomía. 


\section{Conclusiones}

En relación con el primer objetivo, el estudio ha encontrado una correspondencia media-baja entre los temas prioritarios para los ciudadanos según datos demoscópicos y los temas que se tratan en Twitter. Dado que poco más del $20 \%$ de los tuits incluyen menciones a temas clave, se concluye que las preocupaciones expresadas en Twitter no son representativas de los intereses del conjunto de la sociedad. Esta discrepancia entre el mundo online y el mundo offline apunta a la creación de guetos políticos en Twitter con participantes, habitualmente más politizados que el resto de la sociedad, que retroalimentan sus propios temas con escasa conexión con el resto de la sociedad (Pedro y Fenton, 2017).

También conviene tener en cuenta que la mayor parte de los usuarios de Twitter tienen un perfil joven, lo que puede afectar a la selección de temas (por ejemplo, muchos jóvenes no se han visto afectados negativamente por el sistema de salud). La desconexión respecto a los temas prioritarios en las encuestas puede verse impulsada también por la exigencia de brevedad y, por tanto, superficialidad, de la red social Twitter, en donde activistas políticos y opinadores suelen concentrar su atención en dos o máximo tres temas (Trillos y Soto, 2018).

Otro factor que se debe considerar es que Twitter es utilizado como herramienta de campaña de forma consciente para conseguir algún tipo de fin, con los tuiteros llevando la iniciativa en la selección de los temas. Sin embargo, la encuesta es cerrada y no ofrece posibilidad de acción proactiva por parte del encuestado, donde este es un sujeto pasivo.

Por último, hay que tener en cuenta que los medios de comunicación tradicionales siguen teniendo una influencia muy importante en las sociedades y, a menudo, son capaces de "imponer su agenda" en la opinión pública a través de sus ediciones analógicas y digitales (Trillos y Soto 2018, p. 71). En definitiva, el perfil de usuarios de Twitter, las características de las herramientas y la influencia de los medios de masas en la sociedad favorecen que los discursos en Twitter difieran sustancialmente de los que se consiguen con una encuesta de ámbito nacional.

Esta conclusión puede ser matizada por tres hallazgos. En primer lugar, por la presencia media-alta de TT que incluyen al menos un tuit de alguno de los temas prioritarios. En segundo lugar, por una correspondencia media-alta entre el orden de importancia de los temas prioritarios para los ciudadanos y el orden de importancia de estos temas en Twitter, excepto en salud, que es el tema principal en las encuestas y el último de los cinco en Twitter. En tercer lugar, por el dominio de valores vinculados a la equidad, además del empleo de tonos y evaluaciones positivas relacionadas con la mejora de las condiciones socioeconómicas de los ciudadanos. Si bien, estos datos sugieren un interés de los tuiteros por favorecer a la mayoría de la población mediante el consenso, el hecho es que los debates en Twitter no están dominados por los temas concretos que los ciudadanos consideran de mayor importancia (Jungherr et al., 2017).

Respecto al segundo objetivo, se ha encontrado que la mayor parte de los emisores tienen una ideología de centro, superando en 12 puntos a la izquierda y en 15 a la derecha. Sin embargo, el centro solamente tiene la hegemonía en uno de los temas (educación), mientras que la derecha y la izquierda hegemonizan dos temas cada uno (desempleo e inseguridad/ salud y corrupción, respectivamente). Se deduce que la derecha, seguida de la izquierda, han sido capaces de hegemonizar más temas con menos tuits. La eficacia global del centro se ve reducida por concentrar sus esfuerzos en un solo tema. En términos generales, puede afirmarse que las relaciones de poder por la hegemonía en Twitter son equilibradas.

Respecto a las narrativas concretas, se observa que la conceptualización realizada por Wallerstein (2001) de las tres ideologías dominantes de la modernidad en función de su relación con la velocidad del cambio aplican a las narrativas de izquierda y centro, pero no así a las de derecha. La retórica de izquierda vinculada a Gustavo Petro propone acelerar el cambio sociopolítico. Sin embargo, cabe señalar que, si bien se proponen cambios profundos, no se trata de revolución, sino de reforma. La de centro plantea que Sergio Fajardo gestionará el cambio responsablemente y con moderación. Por otro lado, la retórica de derecha vinculada a Iván Duque no 
aboga por preservar el statu quo \#como sería característico del conservadurismo\#, sino que, estratégicamente, se posiciona a favor del cambio moderado en oposición al gobierno anterior (también de derecha) de Juan Manuel Santos.

Otro aspecto de interés que Wallerstein (2001) considera importante en el análisis de las ideologías, es el actor social aliado de cada corriente. Se observa continuidad respecto a la modernidad en la derecha, que no identifica aliados importantes en la sociedad civil, pues su programa se llevará a cabo desde arriba por el propio Gobierno y sus aliados empresariales. También hay continuidad en el centro, pues se alía con la población educada y honrada para que las élites intelectuales (gubernamentales y académicas) gestionen el cambio. La diferencia se encuentra en la izquierda, puesto que la clase obrera \# actor principal del cambio de izquierdas durante la modernidad\# no aparece como sujeto clave. Hay algunas referencias a la comunidad educativa, además de menciones escasas a ciudadanos y jóvenes. En este sentido, la narrativa de izquierda deja de lado el discurso de la lucha de clases, pero no identifica con claridad ni trata de conformar el sujeto concreto del cambio.

Las relaciones con otros actores que se establecen en los tuits se centran prioritariamente en los adversarios políticos. La izquierda se opone, especialmente, a la derecha que representa al establishment y, en segundo lugar, al centro por proponer medidas superficiales. Por tanto, sus relatos enfatizan contra quién están, pero no a favor de quiénes están de una manera concreta. Esta dinámica es aún más acuciante en la derecha, que enfatiza estratégicamente su lucha contra los peligros del populismo "castro-chavista" atribuido a Petro. En menor medida, la derecha vinculada a Duque también construye discursivamente al gobierno de Santos y su sucesor, Vargas Lleras, como adversarios, para posicionarse como una fuerza de cambio. El centro se opone en igual medida al populismo de Petro y a la corrupción de la derecha.

También resulta pertinente la distinción realizada por Bobbio (2004) entre izquierda y derecha. Se confirma que la izquierda antepone la igualdad al crecimiento y que la derecha antepone el crecimiento a la igualdad, si bien ninguna de las dos ideologías excluye la dimensión secundaria. Se observa que el discurso de centro trata de otorgar la misma importancia a ambas dimensiones.

Se ha encontrado que la diferenciación propuesta por Galtung (1992) respecto a la priorización del mercado o del Estado en el funcionamiento de la economía aplica a las tres ideologías analizadas. La derecha aboga por más mercado, la izquierda por más Estado en un contexto histórico de neoliberalismo (pero sin pretender eliminar el mercado, sino con la construcción de un Estado protector desarrollista) y el centro por combinar ambos, aunque con mayor fuerza por parte del mercado. Se observa que la derecha promueve más neoliberalismo, mientras que la izquierda y el centro se acercan a posiciones socialdemócratas, con la izquierda adoptando una postura más oposicional con el neoliberalismo.

Por último, cabe dejar anotado que el estudio se ha centrado en el nivel del discurso y que en el ámbito de las prácticas políticas puede haber consonancia o disonancia con el discurso. La función de los ciudadanos críticos es siempre mantener el escepticismo respecto al discurso y comparar ambos niveles para extraer sus propias conclusiones.

\section{Agradecimientos}

Los autores quieren agradecer a Miguel Ángel Andreu Segura, Nataly Insuasty Sánchez y Bibiana Moreno su colaboración en la recogida de datos de análisis de contenido.

\section{Referencias}

Bobbio, N. (2004). Destra e sinistra. Donzelli Editore. 
Bonilla, J., y Velandia, C. (2010). Proceso electoral colombiano de 2010: entre el ajuste y la consolidación del uribismo. Comentario Internacional. Revista del Centro Andino de Estudios Internacionales, 10, 17-46. https://revistas.ua sb.edu.ec/index.php/comentario/article/view/87

Caldevilla, D., Rodríguez-Terceño, J., y Barrientos, A. (2019). El malestar social a través de las nuevas tecnologías: Twitter como herramienta política. Revista Latina de Comunicación Social, 74, 1264-1290. https://doi.org/10 $.4185 /$ RLCS-2019-1383

Campos-Domínguez, E. (2017). Twitter y la comunicación política. El Profesional de la Información, 26(5), 785-793. https://doi.org/10.3145/epi.2017.sep.01

Castromil, A., Rodríguez-Díaz, R., y Garrigós, P. (2020). La agenda política en las elecciones de abril de 2019 en España: programas electorales, visibilidad en Twitter y debates electorales. El Profesional de la Información, 29(2), e290217. https://doi.org/10.3145/epi.2020.mar.17

Cifuentes, C. F., y Pino, J. (2018). Conmigo o contra mí: análisis de la concordancia y estrategias temáticas del Centro Democrático en Twitter. Palabra Clave, 21(3), 885-916. https://doi.org/10.5294/pacla.2018.21.3.10

Correa, J., y Camargo, J. (2017). Ideological consumerism in Colombian elections, 2015: Links between political ideology, Twitter activity, and electoral results. Cyberpsychology, Behavior, and Social Networking, 20(1), 37-43. https://doi.org/10.1089/cyber.2016.0402

El Tiempo. (2 de septiembre de 2017). ¿Cometieron las Farc un error al mantener siglas en su nuevo partido? El Tiempo. https://www.eltiempo.com/politica/partidos-politicos/significado-del-logo-y-el-nombre-del-partidopolitico-de-las-farc-126066

Fernández, C. (2012). Twitter y la ciberpolítica. Anuario Electrónico de Estudios en Comunicación Social. Disertaciones, 5(1), 9-24. https://revistas.urosario.edu.co/index.php/disertaciones/article/view/3884

Gallego, J., Munger, K., Martínez, J., y Vásquez, M. (2017). Tweeting for peace: Experimental evidence from the 2016 Colombian plebiscite (Documento de trabajo N. 015852). Universidad del Rosario. https://ideas.repec.org/p/ $\mathrm{col} / 000092 / 015852 . \mathrm{html}$

Galtung, J. (1992). The way is the goal: Gandhi today. Gujarat Vidyapith.

García-Dussán, E. (2011). El delirio mockusiano: tecnologías de la comunicación y acción política juvenil. Revista Tendencias \& Retos, 16, 291-305. https://dialnet.unirioja.es/servlet/articulo?codigo $=4929345$

García-Perdomo, V. (2017). Between peace and hate: Framing the 2014 Colombian presidential election on Twitter. Cuadernos.info, 41, 57-70. https://doi.org/10.7764/cdi.41.1241

Gomes, W., Fernandes, B., Reis, L., y Silva, T. (2009). The election campaign on line of Barack Obama in 2008. Cuadernos de H Ideas, 3(3). http://sedici.unlp.edu.ar/handle/10915/33287

Gramsci, A. (2004). Antología.selección, traducción y notas de Manuel Sacristán. Siglo XXI.

Investigación y Asesoría de Mercadeo [Invamer]. (2018). Elecciones 2018. Medición \#7. Mayo 2018. https://static.iris .net.co/semana/upload/documents/gran-encuesta-mayo-17.pdf

Jara, R., Faure, A., Beltrán, J., y Castro, G. (2017). La notoriedad política en los candidatos que usan Twitter. Un ejercicio de clusterización durante las elecciones municipales en Chile (2016). Revista Latina de Comunicación Social, 72, 803-817. https://doi.org/10.4185/RLCS-2017-1193

Jungherr, A., Schoen, H., and Jürgens, P. (2015). The mediation of politics through Twitter: An analysis of messages posted during the campaign for the German Federal Election 2013. Journal of Computer-Mediated Communication, 21(1), 50-68. https://doi.org/10.1111/jcc4.12143

Jungherr, A., Schoen, H., Posegga, O., \& Jürgens, P. (2017). Digital trace data in the study of public opinion: An indicator of attention toward politics rather than political support. Social Science Computer Review, 35(3), 336-356. https://doi.org/10.1177/0894439316631043

Larsson, A. O., y Moe, H. (2012). Studying political microblogging: Twitter users in the 2010 Swedish election campaign. New Media \& Society, 14(5), 729-747. https://doi.org/10.1177/1461444811422894 
López-Trigo, M., García, R., y Femenia, S. (2013). La comunicación política en los “Social media”: análisis comparado de la campaña de Barack Obama y Hillary Clinton en 2008 [Número especial]. Historia y Comunicación Social, 18, 813-826. https://doi.org/10.5209/rev HICS.2013.v18.44367

Martín Serrano, M. (2004). La producción social de comunicación. Alianza Editorial.

Mejía Cortés, J. (2018). Comunicación política y posverdad: expresiones discursivas de la oposición al plebiscito en Colombia 2016. Diálogos de Derecho y Política, 20, 8-41. https://revistas.udea.edu.co/index.php/derypol/artic le/view/332509

Pedro, J., y Fenton, N. (2017). Una conversación con Natalie Fenton: resocializar la política y repolitizar la economía. deSignis, 26, 207-223. http://www.designisfels.net/publicaciones/revistas/26.pdf

Percastre-Mendizábal, S., Pont-Sorribes, C., \& Codina, L. (2017). A sample design proposal for the analysis of Twitter in political communication. El profesional de la Información, 26(4), 579-588. https://doi.org/10.3145/epi.201 7.jul.02

Quintero Giraldo, Y. (2018). Interacción a partir de los mensajes sobre corrupción publicados en Twitter por los precandidatos a la presidencia de Colombia. Revista Colombiana de Ciencias Sociales, 9(2), 440-476. https://d oi.org/10.21501/22161201.2618

Richard, E. (2011). Storytelling, narrativas de campaña. Campañas electorales para la presidencia en Colombia, 2010. OPERA, 11(11), 129-145. https://revistas.uexternado.edu.co/index.php/opera/article/view/3567

Rincón, O. (2011). Mucho ciberactivismo... pocos votos: Antanas Mockus y el Partido Verde colombiano. Nueva Sociedad, 235, 74-89. https://biblat.unam.mx/es/revista/nueva-sociedad/articulo/mucho-ciberactivismo-poco s-votos-antanas-mockus-y-el-partido-verde-colombiano

Said-Hung, E. y Arcila, C. (2011). Líderes de opinión en Colombia, Venezuela e Irán. El caso de los 20 usuarios más vistos en Twitter. Comunicación y Sociedad, 14(1), 75-100. https://core.ac.uk/download/pdf/83574301.pdf

Segado-Boj, F., Díaz-Campo, J., y Lloves-Sobrado, B. (2015). Líderes latinoamericanos en Twitter. Viejas costumbres para nuevos medios en tiempos de crisis políticas. Revista Latina de Comunicación Social, 70, 156-173. https:/ /doi.org/10.4185/RLCS-2015-1040

$\mathrm{Su}, \mathrm{Y}$., y Borah, P. (2019). Who is the agenda setter? Examining the intermedia agenda-setting effect between Twitter and newspapers. Journal of Information Technology \& Politics, 16(3), 236-249. https://doi.org/10.1080/19331 681.2019 .1641451

Trillos, J., y Soto, J. (2018). El poder de los medios masivos tradicionales y las plataformas digitales en el activismo político. Revista Encuentros, 16, 62-78. https://doi.org/10.15665/encuent.v16i02.1440

Wallerstein, I. (2001). Después del liberalismo. Siglo XXI.

YanHaas. Advanced Market Research. (2018). La gran encuesta. Elecciones 2018. Elecciones presidenciales 2018. https ://imgcdn.larepublica.co/cms/2018/05/17185339/Gran-Encuesta-YanHaas.pdf

\section{Notas}

* Artículo de investigación científica. La investigación de la cual se deriva el presente artículo ha sido financiada por el Programa Estatal de I+D+i Problemas públicos y controversias: diversidad y participación en la esfera mediática (CSO2017-82109-R) (España) y por el Fondo para el Desarrollo de la Investigación, de la Universidad Santo Tomás, código 20480010 (Colombia).

1 El nombre que ahora significa Fuerza Alternativa Revolucionaria del Común fue elegido tras un congreso realizado en 2017 donde participaron 1200 excombatientes de las FARC ( El Tiempo, 2017).

2 Partido político creado por Álvaro Uribe Vélez en 2013, que nació producto del distanciamiento con las políticas de gobierno de Juan Manuel Santos en su primer periodo presidencial (2010-2014). Uno de los puntos claves fue la decisión del Gobierno de iniciar negociaciones de paz con las FARC-EP (Fuerzas Armadas Revolucionarias de Colombia-Ejército del Pueblo).

3 La firma encuestadora fue contratada por la agrupación de medios radiales, televisivos e impresos que se señalan a continuación: RCN Radio, LaFM, RCN Televisión, El País, El Universal, El Colombiano, Vanguardia Liberal, La República. 
4 La firma encuestadora fue contratada por los medios de información revista Semana, Caracol Televisión y Blu Radio.

$5 \mathrm{Al}$ asignar valores al ranking de temas identificados (a menor valor al sumar ambas columnas, mayor su nivel de importancia), salud (2) se erige como el tema más potente al estar en la cabecera de ambos estudios demoscópicos, seguido algo distanciado de educación (6), desempleo (7), corrupción (8) y seguridad (8).

6 TT correspondiente al 16 de abril de 2018 en el que el periodista Juan Diego Alvira entrevistó al candidato Gustavo Petro en la emisión de la mañana de Caracol Noticias sobre temas de seguridad.

7 en los medios masivos de información colombianos, forma como se le denomina al rechazo de la atención médica a un paciente, por pertenecer a una EPS deudora, lo que decanta en el traslado de la persona de un hospital a otro esperando ser atendido.

\section{Licencia Creative Commons CC BY 4.0}

Para citar este articulo: Alvarado-Vivas, S., López López, J., y Pedro-Carañana, J. (2020). Los debates electorales en Twitter y su correspondencia con las preocupaciones ciudadanas en la contienda presidencial en Colombia 2018. Signo y Pensamiento, 39. https://doi.org/10.11144/Javeriana.syp39.detc 